\title{
Production and physico-chemical quality of wine from mango
}

\author{
Lalit Kumar*1, Samsher ${ }^{1}$, Suresh Chandra ${ }^{1}$, GR Singh ${ }^{1}$, Vaishali ${ }^{2}$, Puroshottam ${ }^{3}$, and \\ Akash Tomar ${ }^{4}$ \\ ${ }^{1}$ Department of Agricultural Engineering and Food Technology \\ ${ }^{2}$ Department of Ag. Biotechnology \\ ${ }^{3}$ Department of Microbiology \\ ${ }^{4}$ Department of Recombinant DNA Technology \\ SVP University of Agriculture and Technology, Meerut (U.P.) 250110 \\ *Corresponding Author: biotechlalit@gmail.com
}

\begin{abstract}
Mango, "The King of Fruits" is known for its taste, flavor, attractive color and nutritional value. India is the major producer of mango among the countries in the world. This particular fruit is not exploited fully despite the fact that it has a very good sugar content and antioxidant activity. Because of the sugar content present, mango juice is a very good substrate for fermentation. In order to prevent the post harvest loss, mango juice can be used for wine production. Three mango cultivars which are commonly found in the region were selected for the study. Optimization of fermentation conditions (like yeast strain, pectinase enzyme and inoculum concentration) and production of higher alcohols and other physicochemical characteristics during wine fermentation were investigated. Mango juice of Dasheheri, Chausa and Gulab Gamun was divided into 3 parts, which contained an inoculum concentration of $5 \%, 10 \%$, and $15 \%$, two yeast strains( IARI and native) for each and the $\mathrm{pH}$ was adjusted to 4.0 and sugar concentration was adjusted to $20^{\circ} \mathrm{B}$ by adding sugar solution. The variety Chausa recorded highest TSS content $\left(12^{\circ} \mathrm{B}\right)$ followed by the varieties Dasheheri $\left(11^{\circ} \mathrm{B}\right)$ and Gulab jamun $\left(10^{\circ} \mathrm{B}\right)$. The higher value of ethanol production was found $10.70 \mathrm{v} / \mathrm{v}$ of the sample of chausa having $15 \%$ inoculum concentration and IARI strain after 30 days. The lowest value of ethanol production was observed $4.60 \mathrm{v} / \mathrm{v}$ of the sample of gulab jamun having 5\% inoculum concentration with both strains after 60 days.
\end{abstract}

Keywords: Fermentation, Production, physico-chemical, mango, wine.

Paper cited: Kumar, L., Samsher; Chandra, S., Singh, G.R., Vaishali; Puroshottam; and Tomar, A. (2016). Production and physico-chemical quality of wine from mango. South Asian Journal of Food technology and Environment, 2(1): 321-329.

Received: 20/01/2016 Revised: 25/02/2016 Accepted: 24/02/2016

\section{Introduction}

The mango is a juicy stone fruit belonging to the genus Mangifera, that are cultivated mostly for edible fruit. Mango is one of the most highly priced desert fruits of the tropics. It has rich luscious, aromatic flavour and a delicious taste in which sweetness and acidity is delightfully blended. Mango production has experienced continuous growth in the last decades of the $20^{\text {th }}$ century (Baisya, 2004).

In developing countries like India 20$30 \%$ of fruits produced are wasted due to lack of proper utilization, post-harvest and processing technology. By converting the waste into value added products like wine is a smart solution for this problem. There are few reports on the suitability of mango for wine production. Strains of Saccharomyces cerevisiae yeast which are known to produce different volatile profiles have been commonly used for alcoholic fermentation. But there is lack of information on mango wine production especially dealing with the suitability of local mango cultivars for wine production, other yeast strains, optimization of conditions of fermentation and characterization of its wine produced (both chemical and physical factors for quality). Accordingly, these aspects were investigated and the results are reported in this communication.

Mangoes are widely used in cuisine. Sour, unripe mangoes are used in chutneys, athanu, pickles, side dishes, or 
may be eaten raw with salt, chili, or soy sauce. A summer drink called Aam panna comes from mangoes. Mango pulp made into jelly or cooked with red gram dhal and green chillies may be served with cooked rice. Mango lassi is popular throughout South Asia, prepared by mixing ripe mangoes or mango pulp with buttermilk and sugar. Ripe mangoes are also used to make curries. Aamras is a popular thick juice made of mangoes with sugar or milk, and is consumed with chapatis or pooris. The pulp from ripe mangoes is also used to make jam called mangada.

Wine making is one of the most ancient technologies and is now one of the most commercially prosperous biotechnological processes. Even though the grapes are the main raw material used for the wine production, there is an increasing interest in the search for indigenous fruits such as orange, apple, mango, and also palm sap that are cheap and readily available for wine making in such countries where grapes are not abundantly available (Reddy and Reddy, 2005). However the production of wine from mango, which has a high carbohydrate content $(16-18 \% \mathrm{w} / \mathrm{v})$, is one of the alternative ways to exploit and convert the surplus production into a valuable product (Kumar et al., 2009), and it has been proved that mango wine contains bioactive molecules which impart antioxidant activity to the wine (Varakumar et al., 2011).

Optimum $\mathrm{pH}$ value is necessary for yeast growth and ethanol production. Most of the yeasts grow very well between $\mathrm{pH} 4.5$ and 6.5 and nearly all species are not able to grow in more acidic or alkaline media. Low or high $\mathrm{pH}$ values are known to cause chemical stress on yeast cell (Walker, 1998). $\mathrm{SO}_{2}$ is used as preservative agent in wine because it has several functions like preventing enzymatic oxidation of the must and inhibits the growth of undesirable microorganisms in wine preservation. At present, it is difficult to make quality wines without the addition of $\mathrm{SO}_{2}$ as the sulfur is an essential component for yeast growth. (Bafrncova et al., 1999). Very few systematic studies are reported on the effect of temperature, $\mathrm{pH}, \mathrm{SO}_{2}$ and aeration on yeast growth and quality other than wine from grapes. Hence, these factors must be studied with more detail, especially their influence on microorganisms and quality of wine produce from fruits.

There has been limited information in the research on mango wine until recently, although it started from 1960's. Czyhrinciwk (1966) reported the first study on mango wine production. Onkarayya and Singh (1984) screened twenty varieties of mangoes from India for wine production. Reddy and Reddy (2005) developed a method of mango juice extraction with pectinase and characterized ethanol and some volatile contents of mango wine.

\section{Materials and Methods}

Fruits Collection: Mango fruits were procured from the Department of Horticulture and local market, Meerut (UP), India. The mango varieties namely Dashehari, GulabJamun and Chausa were selected and used for the study. Healthy and ripe fruits were selected after manual sorting and discarding the defective fruits.

Pulp Extraction: Ripened mango fruits were washed in hot water and peeled manually using knife. The flesh was cut away from the seed using knife and then homogenized with the help of pulp extractor.

Juice Preparation: After pulp extraction, Juice was obtained by passing the pulp through a muslin cloth and then centrifuged at $5000 \mathrm{rpm}$ for 10 minutes. Juice obtained in this manner was then subjected to physicochemical analysis. The juice was pasteurized at $60^{\circ} \mathrm{C}$ for 30 minutes and cooled immediately with running tap water. The $\mathrm{pH}$ of the mango juice was adjusted to 4.0 by addition of calcium carbornate and citric acid respectively.

Yeast Strain: Yeast strain (Saccharomyces Cerevisiae 1035) was procured from Indian Type Culture Collection (ITCC), Division of Plant Pathology, Indian Agricultural Research Institute (IARI), Pusa, New Delhi.

Broth Preparation: Thirty gram YPD mix well with $600 \mathrm{ml}$ distilled and heated for 5 minute. $100 \mathrm{ml}$ broth poured in $250 \mathrm{ml}$ conical flask and then autoclaved.

Preparation of Sugar Solution: Granulated sucrose was procured from the local market. The sugar solution was prepared by dissolving $250 \mathrm{~g}$ granulated sucrose in boiled $250 \mathrm{ml}$ of water for 10 minutes and then allowed to cool at room temperature and stored aseptically. 
Fermentation of the Mango Juice: The pre-treated mango juice was divided into different portions of $200 \mathrm{ml}$ and transferred it in to $500 \mathrm{ml}$ sterilized Erlenmeyer conical flasks. The inoculum concentration of $5 \%$, $10 \%$ and $15 \%$ was transferred in each $500 \mathrm{ml}$ conical flasks having $200 \mathrm{ml}$ mango juice. The flasks were closed using a cotton plug and polyethene with a bend of thread. These were allowed for fermentation for 15 days. The experiments were carried out at room temperature. Fermentation rate was monitored every 24 hours by checking the ${ }^{\circ} \mathrm{Bx}$ change. End of fermentation was determined when the ${ }^{\circ} \mathrm{Bx}$ could not change any further. After fermentation, the wine samples were filtered (vacuum filter using $0.2 \mu \mathrm{m}$ filter paper) prior to analysis. All the determinations were done in triplicates and the mean values determined. The clear supernatant samples were kept in refrigerator for a few weeks until the physicochemical analyses were completed. At the end of fermentation, the wines were stabilized with the addition of KMS @ 85 ppm and preserved.

\section{Results and Discussion}

Effect on pH: The $\mathrm{pH}$ of mango wine showed a decreasing trend for all the treatments with storage period up to 60 days (Table 1). The $\mathrm{pH}$ of the samples of three different mango varieties having inoculum concentrations of $5 \%, 10 \%$ and $15 \%$ with two different strains namely IARI and native were measured as 4.5 , respectively in the fresh samples. The lowest value of $\mathrm{pH}$ (3.69) was observed for the sample of Dasheheri having 15\% inoculum concentration with native strain and highest value of $\mathrm{pH}$ (3.85) was observed for the sample of Chausa having $5 \%$ inoculum concentration and IARI strain after 60 days. However, in general the lowest value of $\mathrm{pH}$ was observed after 60 days irrespective of varieties, inoculum concentrations and strains.

Effect on Total Soluble Solid (TSS): The study revealed that TSS of the samples having inoculum concentrations of $5 \%, 10 \%$ and $15 \%$ with two different strains namely IARI (Saccharomyces Cerevisiae 1035) and native were observed as $20{ }^{\circ} \mathrm{Brix}$ in fresh samples. The TSS values of all the wine samples of Dashari, Chausa and Gulab Jamun having inoculum concentration of $5 \%, 10 \%$ and $15 \%$ with IARI (S. Cerevisiae 1035) and native strain were observed as $0{ }^{0} \mathrm{Brix}$ in refrigerator at $10^{\circ} \mathrm{C}$ after 60 days of storage period (Table 2).

Effect on Acidity: The acidity of mango wine increased with increasing the storage period upto 60 days among all the samples. The lowest value of acidity (1.510) was observed for the sample of Dasheheri having 5\% inoculum concentration with IARI strain and highest value of acidity (1.944) was observed for the sample of Gulab Jamun having 15\% inoculum concentration with both strains after 60 days. However, in general higher value of acidity was observed after 60 days irrespective of varieties, inoculum concentrations and strains (Table 3).

Effect on Ascorbic Acid: The ascorbic acid of mango wine showed decreasing trend for all the treatments with storage period up to 60 days. The lowest value of ascorbic acid (11.18) was observed for the sample of Chausa having 5\% inoculum concentration with IARI strain and highest value of ascorbic acid (20.60) was observed for the sample of Dasheheri having 5\% inoculum concentration and both strain after 60 days (Table 4).

Effect on Ethanol: The ethanol production of mango wine showed an increasing trend for all the treatments with storage period up to 30 days and after 30 days showed decreasing order for all the treatments up to 60 days. The lowest value of ethanol production (4.60) was observed for the sample of Gulab Jamun having 5\% inoculum concentration with both strains after 60 days and highest value of ethanol production (10.70) was observed for the sample of Chausa having 15\% inoculum concentration with IARI strain after 30 days. The ethanol production found best in Chausa followed by Dasheheri and Gulab Jamun after 30 days of storage (Table 5).

\section{Conclusion}

Based on these studies, it can be concluded that Dashehri, Chausa and Gulab Jamun cultivars are most suitable for mango wine production. Therefore, as mango production is tremendously highest in India, their use in wine production would greatly reduce the cases of post harvest losses and thus go a long way in contributing considerably to the economy of not only India but also international mango producers. 
More so, information on adequate process for both juice and wine production from mango and other tropical fruits can be of valuable reference to the wine industry. As per ethanol production, the Chausa variety was found most suitable for wine production among all the other varieties.

Table 1: Effect of inoculum concentrations, strains and storage period on pH of mango wine samples

\begin{tabular}{|c|c|c|c|c|c|c|}
\hline \multirow{3}{*}{$\begin{array}{l}\text { Storage } \\
\text { Period } \\
\text { (Days) }\end{array}$} & \multicolumn{6}{|c|}{ Inoculum Concentration (5\%) } \\
\hline & \multicolumn{3}{|c|}{ IARI Strain } & \multicolumn{3}{|c|}{ IARI Strain } \\
\hline & $\mathbf{D}$ & $\mathbf{C}$ & $\mathbf{G}$ & $\mathbf{D}$ & $\mathbf{C}$ & $\mathbf{G}$ \\
\hline 0 & 4.50 & 4.50 & 4.50 & 4.50 & 4.50 & 4.50 \\
\hline 15 & 4.35 & 4.34 & 4.34 & 4.34 & 4.35 & 4.34 \\
\hline 30 & 4.20 & 4.21 & 4.21 & 4.22 & 4.20 & 4.21 \\
\hline 45 & 4.00 & 4.05 & 4.04 & 4.02 & 4.04 & 4.03 \\
\hline 60 & 3.80 & 3.85 & 3.82 & 3.78 & 3.83 & 3.80 \\
\hline $\mathrm{CD}$ & 0.012 & 0.015 & 0.013 & 0.013 & 0.013 & 0.010 \\
\hline SE(d) & 0.005 & 0.007 & 0.006 & 0.006 & 0.006 & 0.004 \\
\hline $\mathrm{SE}(\mathrm{m})$ & 0.004 & 0.005 & 0.004 & 0.004 & 0.004 & 0.003 \\
\hline $\mathrm{CV}$ & 0.152 & 0.195 & 0.175 & 0.175 & 0.174 & 0.124 \\
\hline \multirow{3}{*}{$\begin{array}{l}\text { Storage } \\
\text { Period } \\
\text { (Days) }\end{array}$} & \multicolumn{6}{|c|}{ Inoculum Concentration (10 \%) } \\
\hline & \multicolumn{3}{|c|}{ IARI Strain } & \multicolumn{3}{|c|}{ Native Strain } \\
\hline & D & $\mathrm{C}$ & $\mathbf{G}$ & D & $\mathrm{C}$ & G \\
\hline 0 & 4.50 & 4.50 & 4.50 & 4.50 & 4.50 & 4.50 \\
\hline 15 & 4.29 & 4.31 & 4.29 & 4.32 & 4.32 & 4.30 \\
\hline 30 & 4.19 & 4.17 & 4.19 & 4.16 & 4.17 & 4.18 \\
\hline 45 & 3.99 & 4.03 & 4.00 & 4.01 & 4.03 & 4.02 \\
\hline 60 & 3.75 & 3.77 & 3.81 & 3.74 & 3.77 & 3.80 \\
\hline $\mathrm{CD}$ & 0.014 & 0.013 & 0.012 & 0.010 & 0.013 & 0.012 \\
\hline SE(d) & 0.006 & 0.006 & 0.005 & 0.004 & 0.006 & 0.005 \\
\hline $\mathrm{SE}(\mathrm{m})$ & 0.004 & 0.004 & 0.004 & 0.003 & 0.004 & 0.004 \\
\hline $\mathrm{CV}$ & 0.187 & 0.176 & 0.152 & 0.124 & 0.176 & 0.152 \\
\hline \multirow{3}{*}{$\begin{array}{l}\text { Storage } \\
\text { Period } \\
\text { (Days) }\end{array}$} & \multicolumn{6}{|c|}{ Inoculum Concentration (15\%) } \\
\hline & \multicolumn{3}{|c|}{ IARI Strain } & \multicolumn{3}{|c|}{ IARI Strain } \\
\hline & $\mathbf{D}$ & $\mathrm{C}$ & $\mathbf{G}$ & $\mathbf{D}$ & $\mathrm{C}$ & G \\
\hline 0 & 4.50 & 4.50 & 4.50 & 4.50 & 4.50 & 4.50 \\
\hline 15 & 4.31 & 4.31 & 4.31 & 4.31 & 4.31 & 4.31 \\
\hline 30 & 4.16 & 4.16 & 4.16 & 4.16 & 4.16 & 4.16 \\
\hline 45 & 4.00 & 4.00 & 4.00 & 4.00 & 4.00 & 4.00 \\
\hline 60 & 3.71 & 3.71 & 3.71 & 3.71 & 3.71 & 3.71 \\
\hline $\mathrm{CD}$ & 0.012 & 0.012 & 0.012 & 0.012 & 0.012 & 0.012 \\
\hline $\mathrm{SE}(\mathrm{d})$ & 0.005 & 0.005 & 0.005 & 0.005 & 0.005 & 0.005 \\
\hline $\mathrm{SE}(\mathrm{m})$ & 0.004 & 0.004 & 0.004 & 0.004 & 0.004 & 0.004 \\
\hline $\mathrm{CV}$ & 0.153 & 0.153 & 0.153 & 0.153 & 0.153 & 0.153 \\
\hline
\end{tabular}

Where, $\mathrm{D}=$ Dasheheri, $\mathrm{C}=$ Chausa, $\mathrm{G}=$ Gulab Jamun 
Table 2: Effect of inoculum concentrations, strains and storage period on total soluble solid ( ${ }^{0}$ Brix) of mango wine samples

\begin{tabular}{|c|c|c|c|c|c|c|}
\hline \multirow{3}{*}{$\begin{array}{l}\text { Storage } \\
\text { Period } \\
\text { (Days) }\end{array}$} & \multicolumn{6}{|c|}{ Inoculum Concentration (5\%) } \\
\hline & \multicolumn{3}{|c|}{ IARI Strain } & \multicolumn{3}{|c|}{ IARI Strain } \\
\hline & $\mathbf{D}$ & $\mathbf{C}$ & $\mathbf{G}$ & D & $\mathbf{C}$ & $\mathbf{G}$ \\
\hline 0 & 20.00 & 20.00 & 20.00 & 20.00 & 20.00 & 20.00 \\
\hline 15 & 15.50 & 15.00 & 14.83 & 15.50 & 15.00 & 14.83 \\
\hline 30 & 9.66 & 9.50 & 9.25 & 9.66 & 9.30 & 9.33 \\
\hline 45 & 1.16 & 1.00 & 1.92 & 1.16 & 1.00 & 1.00 \\
\hline 60 & 00.00 & 00.00 & 00.00 & 00.00 & 00.00 & 00.00 \\
\hline $\mathrm{CD}$ & 0.981 & 0.921 & 0.789 & 0.981 & 0.921 & 0.789 \\
\hline $\mathrm{SE}(\mathrm{d})$ & 0.435 & 0.408 & 0.350 & 0.435 & 0.408 & 0.350 \\
\hline $\mathrm{SE}(\mathrm{m})$ & 0.307 & 0.289 & 0.247 & 0.307 & 0.289 & 0.247 \\
\hline $\mathrm{CV}$ & 5.744 & 5.495 & 4.740 & 5.744 & 5.495 & 4.740 \\
\hline \multirow{3}{*}{$\begin{array}{l}\text { Storage } \\
\text { Period } \\
\text { (Days) }\end{array}$} & \multicolumn{6}{|c|}{ Inoculum Concentration (10 \%) } \\
\hline & \multicolumn{3}{|c|}{ IARI Strain } & \multicolumn{3}{|c|}{ Native Strain } \\
\hline & D & C & G & D & C & G \\
\hline 0 & 20.00 & 20.00 & 20.00 & 20.00 & 20.00 & 20.00 \\
\hline 15 & 13.83 & 13.33 & 13.66 & 14.00 & 13.50 & 14.00 \\
\hline 30 & 8.66 & 8.33 & 8.33 & 8.83 & 8.66 & 8.83 \\
\hline 45 & 0.66 & 0.83 & 0.83 & 0.66 & 0.86 & 0.66 \\
\hline 60 & 00.00 & 00.00 & 00.00 & 00.00 & 00.00 & 00.00 \\
\hline $\mathrm{CD}$ & 0.412 & 0.412 & 0.583 & 1.064 & 0.256 & 0.789 \\
\hline $\mathrm{SE}(\mathrm{d})$ & 0.183 & 0.183 & 0.258 & 0.471 & 0.114 & 0.350 \\
\hline $\mathrm{SE}(\mathrm{m})$ & 0.129 & 0.129 & 0.183 & 0.333 & 0.080 & 0.247 \\
\hline $\mathrm{CV}$ & 2.590 & 2.600 & 3.691 & 6.636 & 1.616 & 4.960 \\
\hline \multirow{3}{*}{$\begin{array}{l}\text { Storage } \\
\text { Period } \\
\text { (Days) }\end{array}$} & \multicolumn{6}{|c|}{ Inoculum Concentration (15\%) } \\
\hline & \multicolumn{3}{|c|}{ IARI Strain } & \multicolumn{3}{|c|}{ IARI Strain } \\
\hline & D & $\mathbf{C}$ & G & D & $\mathbf{C}$ & $\mathbf{G}$ \\
\hline 0 & 20.00 & 20.00 & 20.00 & 20.00 & 20.00 & 20.00 \\
\hline 15 & 12.00 & 11.66 & 11.50 & 11.66 & 11.50 & 11.50 \\
\hline 30 & 6.33 & 6.66 & 6.00 & 6.66 & 7.00 & 6.66 \\
\hline 45 & 0.33 & 00.00 & 00.00 & 00.00 & 00.00 & 00.00 \\
\hline 60 & 00.00 & 00.00 & 00.00 & 00.00 & 00.00 & 00.00 \\
\hline $\mathrm{CD}$ & 0.981 & 0.673 & 0.921 & 0.673 & 0.921 & 0.476 \\
\hline SE(d) & 0.435 & 0.298 & 0.408 & 0.298 & 0.408 & 0.211 \\
\hline $\mathrm{SE}(\mathrm{m})$ & 0.307 & 0.211 & 0.289 & 0.211 & 0.289 & 0.149 \\
\hline $\mathrm{CV}$ & 6.883 & 4.763 & 6.667 & 4.763 & 6.494 & 3.383 \\
\hline
\end{tabular}


Table 3: Effect of inoculum concentrations, strains and storage period on acidity (\%) of mango wine samples

\begin{tabular}{|c|c|c|c|c|c|c|}
\hline \multirow{3}{*}{$\begin{array}{l}\text { Storage } \\
\text { Period } \\
\text { (Days) }\end{array}$} & \multicolumn{6}{|c|}{ Inoculum Concentration (5 \%) } \\
\hline & \multicolumn{3}{|c|}{ IARI Strain } & \multicolumn{3}{|c|}{ IARI Strain } \\
\hline & $\mathbf{D}$ & $\mathbf{C}$ & $\mathbf{G}$ & $\mathbf{D}$ & $\mathbf{C}$ & $\mathbf{G}$ \\
\hline 0 & 0.320 & 0.360 & 0.392 & 0.320 & 0.371 & 0.400 \\
\hline 15 & 0.580 & 0.622 & 0.700 & 0.580 & 0.600 & 0.700 \\
\hline 30 & 0.800 & 0.862 & 0.890 & 0.800 & 0.861 & 0.892 \\
\hline 45 & 1.110 & 1.260 & 1.640 & 1.112 & 1.260 & 1.642 \\
\hline 60 & 1.510 & 1.600 & 1.940 & 1.512 & 1.662 & 1.942 \\
\hline $\mathrm{CD}$ & 0.004 & 0.004 & 0.004 & 0.004 & 0.003 & 0.003 \\
\hline $\mathrm{SE}(\mathrm{d})$ & 0.002 & 0.002 & 0.002 & 0.002 & 0.001 & 0.002 \\
\hline $\mathrm{SE}(\mathrm{m})$ & 0.001 & 0.001 & 0.001 & 0.001 & 0.001 & 0.001 \\
\hline $\mathrm{CV}$ & 0.245 & 0.218 & 0.213 & 0.243 & 0.188 & 0.165 \\
\hline \multirow{3}{*}{$\begin{array}{l}\text { Storage } \\
\text { Period } \\
\text { (Days) }\end{array}$} & \multicolumn{6}{|c|}{ Inoculum Concentration (10 \%) } \\
\hline & \multicolumn{3}{|c|}{ IARI Strain } & \multicolumn{3}{|c|}{ Native Strain } \\
\hline & D & $\mathbf{C}$ & $\mathbf{G}$ & $\mathbf{D}$ & $\mathbf{C}$ & G \\
\hline 0 & 0.321 & 0.370 & 0.392 & 0.332 & 0.374 & 0.400 \\
\hline 15 & 0.590 & 0.624 & 0.700 & 0.582 & 0.610 & 0.710 \\
\hline 30 & 0.810 & 0.864 & 0.892 & 0.802 & 0.862 & 0.894 \\
\hline 45 & 1.112 & 1.262 & 1.642 & 1.112 & 1.261 & 1.642 \\
\hline 60 & 1.512 & 1.610 & 1.942 & 1.513 & 1.664 & 1.943 \\
\hline $\mathrm{CD}$ & 0.004 & 0.004 & 0.004 & 0.003 & 0.004 & 0.004 \\
\hline $\mathrm{SE}(\mathrm{d})$ & 0.002 & 0.002 & 0.002 & 0.002 & 0.002 & 0.002 \\
\hline $\mathrm{SE}(\mathrm{m})$ & 0.001 & 0.001 & 0.001 & 0.001 & 0.001 & 0.001 \\
\hline $\mathrm{CV}$ & 0.257 & 0.222 & 0.175 & 0.219 & 0.204 & 0.174 \\
\hline \multirow{3}{*}{$\begin{array}{l}\text { Storage } \\
\text { Period } \\
\text { (Days) }\end{array}$} & \multicolumn{6}{|c|}{ Inoculum Concentration (15\%) } \\
\hline & \multicolumn{3}{|c|}{ IARI Strain } & \multicolumn{3}{|c|}{ IARI Strain } \\
\hline & D & $\mathbf{C}$ & G & D & $\mathbf{C}$ & G \\
\hline 0 & 0.330 & 0.370 & 0.402 & 0.332 & 0.370 & 0.400 \\
\hline 15 & 0.595 & 0.625 & 0.700 & 0.584 & 0.612 & 0.712 \\
\hline 30 & 0.812 & 0.864 & 0.892 & 0.804 & 0.864 & 0.895 \\
\hline 45 & 1.113 & 1.264 & 1.645 & 1.114 & 1.266 & 1.644 \\
\hline 60 & 1.514 & 1.612 & 1.944 & 1.514 & 1.666 & 1.944 \\
\hline $\mathrm{CD}$ & 0.003 & 0.003 & 0.004 & 0.003 & 0.005 & 0.003 \\
\hline $\mathrm{SE}(\mathrm{d})$ & 0.002 & 0.001 & 0.002 & 0.001 & 0.002 & 0.001 \\
\hline $\mathrm{SE}(\mathrm{m})$ & 0.001 & 0.001 & 0.001 & 0.001 & 0.002 & 0.001 \\
\hline $\mathrm{CV}$ & 0.217 & 0.157 & 0.208 & 0.163 & 0.273 & 0.150 \\
\hline
\end{tabular}


Table 4: Effect of inoculum concentrations, strains and storage period on ascorbic acid $(\mathrm{mg} / 100 \mathrm{ml})$ of mango wine samples

\begin{tabular}{|c|c|c|c|c|c|c|}
\hline \multirow{3}{*}{$\begin{array}{l}\text { Storage } \\
\text { Period } \\
\text { (Days) }\end{array}$} & \multicolumn{6}{|c|}{ Inoculum Concentration (5 \%) } \\
\hline & \multicolumn{3}{|c|}{ IARI Strain } & \multicolumn{3}{|c|}{ IARI Strain } \\
\hline & $\mathbf{D}$ & $\mathbf{C}$ & $\mathbf{G}$ & D & $\mathbf{C}$ & $\mathbf{G}$ \\
\hline 0 & 34.50 & 24.00 & 28.35 & 34.50 & 24.00 & 28.35 \\
\hline 15 & 31.40 & 21.25 & 25.40 & 31.42 & 21.24 & 25.40 \\
\hline 30 & 28.45 & 18.50 & 22.55 & 28.45 & 18.50 & 22.54 \\
\hline 45 & 24.25 & 14.65 & 19.54 & 24.26 & 14.65 & 19.54 \\
\hline 60 & 20.60 & 11.80 & 15.80 & 20.60 & 11.78 & 15.80 \\
\hline CD & 0.118 & 0.187 & 0.170 & 0.136 & 0.155 & 0.170 \\
\hline $\mathrm{SE}(\mathrm{d})$ & 0.052 & 0.083 & 0.075 & 0.060 & 0.069 & 0.075 \\
\hline $\mathrm{SE}(\mathrm{m})$ & 0.037 & 0.059 & 0.053 & 0.043 & 0.049 & 0.053 \\
\hline $\mathrm{CV}$ & 0.231 & 0.562 & 0.412 & 0.265 & 0.466 & 0.412 \\
\hline \multirow{3}{*}{$\begin{array}{c}\text { Storage } \\
\text { Period } \\
\text { (Days) }\end{array}$} & \multicolumn{6}{|c|}{ Inoculum Concentration (10 \%) } \\
\hline & \multicolumn{3}{|c|}{ IARI Strain } & \multicolumn{3}{|c|}{ Native Strain } \\
\hline & $\mathbf{D}$ & $\mathbf{C}$ & $\mathbf{G}$ & $\mathbf{D}$ & $\mathbf{C}$ & G \\
\hline 0 & 34.45 & 24.00 & 28.35 & 34.44 & 24.00 & 28.35 \\
\hline 15 & 31.42 & 21.20 & 25.42 & 31.42 & 21.20 & 25.40 \\
\hline 30 & 28.48 & 18.45 & 22.52 & 28.46 & 18.45 & 22.54 \\
\hline 45 & 24.24 & 14.62 & 19.52 & 24.24 & 14.62 & 19.54 \\
\hline 60 & 20.55 & 11.76 & 15.76 & 20.50 & 11.76 & 15.80 \\
\hline CD & 0.125 & 0.116 & 0.148 & 0.138 & 0.116 & 0.170 \\
\hline $\mathrm{SE}(\mathrm{d})$ & 0.055 & 0.051 & 0.065 & 0.061 & 0.051 & 0.075 \\
\hline $\mathrm{SE}(\mathrm{m})$ & 0.039 & 0.036 & 0.046 & 0.043 & 0.036 & 0.053 \\
\hline $\mathrm{CV}$ & 0.243 & 0.349 & 0.359 & 0.269 & 0.349 & 0.412 \\
\hline \multirow{3}{*}{$\begin{array}{l}\text { Storage } \\
\text { Period } \\
\text { (Days) }\end{array}$} & \multicolumn{6}{|c|}{ Inoculum Concentration (15\%) } \\
\hline & \multicolumn{3}{|c|}{ IARI Strain } & \multicolumn{3}{|c|}{ IARI Strain } \\
\hline & $\bar{D}$ & $\bar{C}$ & $\bar{G}$ & D & $\bar{C}$ & $\bar{G}$ \\
\hline 0 & 34.50 & 24.00 & 28.35 & 34.50 & 24.00 & 28.35 \\
\hline 15 & 31.44 & 21.18 & 25.42 & 31.42 & 21.20 & 25.40 \\
\hline 30 & 28.48 & 18.42 & 22.50 & 28.50 & 18.42 & 22.48 \\
\hline 45 & 24.28 & 14.60 & 19.52 & 24.30 & 14.62 & 19.52 \\
\hline 60 & 20.50 & 11.74 & 15.74 & 20.45 & 11.74 & 15.74 \\
\hline $\mathrm{CD}$ & 0.125 & 0.094 & 0.119 & 0.100 & 0.789 & 0.179 \\
\hline SE(d) & 0.055 & 0.042 & 0.053 & 0.044 & 0.350 & 0.079 \\
\hline $\mathrm{SE}(\mathrm{m})$ & 0.039 & 0.029 & 0.037 & 0.031 & 0.247 & 0.056 \\
\hline $\mathrm{CV}$ & 0.244 & 0.283 & 0.290 & 0.195 & 2.379 & 0.434 \\
\hline
\end{tabular}


Table 5: Effect of inoculum concentrations, strains and storage period on ethanol production $(\%, v / v)$ of mango wine samples

\begin{tabular}{|c|c|c|c|c|c|c|}
\hline \multirow{3}{*}{$\begin{array}{l}\text { Storage } \\
\text { Period } \\
\text { (Days) }\end{array}$} & \multicolumn{6}{|c|}{ Inoculum Concentration (5 \%) } \\
\hline & \multicolumn{3}{|c|}{ IARI Strain } & \multicolumn{3}{|c|}{ IARI Strain } \\
\hline & D & $\mathbf{C}$ & $\mathbf{G}$ & $\mathbf{D}$ & $\mathbf{C}$ & $\mathbf{G}$ \\
\hline 0 & 00.00 & 00.00 & 00.00 & 00.00 & 00.00 & 00.00 \\
\hline 15 & 7.20 & 8.50 & 7.00 & 7.33 & 8.30 & 7.00 \\
\hline 30 & 9.90 & 10.50 & 9.80 & 9.80 & 10.40 & 9.70 \\
\hline 45 & 7.00 & 8.55 & 6.82 & 7.80 & 8.50 & 6.75 \\
\hline 60 & 5.30 & 5.50 & 4.80 & 5.34 & 5.45 & 4.75 \\
\hline $\mathrm{CD}$ & 0.084 & 0.077 & 0.065 & 0.437 & 0.062 & 0.070 \\
\hline $\mathrm{SE}(\mathrm{d})$ & 0.037 & 0.034 & 0.029 & 0.194 & 0.028 & 0.031 \\
\hline $\mathrm{SE}(\mathrm{m})$ & 0.026 & 0.024 & 0.020 & 0.137 & 0.019 & 0.022 \\
\hline $\mathrm{CV}$ & 0.779 & 0.631 & 0.620 & 3.916 & 0.517 & 0.673 \\
\hline \multirow{3}{*}{$\begin{array}{l}\text { Storage } \\
\text { Period } \\
\text { (Days) }\end{array}$} & \multicolumn{6}{|c|}{ Inoculum Concentration (10 \%) } \\
\hline & \multicolumn{3}{|c|}{ IARI Strain } & \multicolumn{3}{|c|}{ Native Strain } \\
\hline & D & $\mathbf{C}$ & $\mathbf{G}$ & D & $\mathbf{C}$ & $\mathbf{G}$ \\
\hline 0 & 00.00 & 00.00 & 00.00 & 00.00 & 00.00 & 00.00 \\
\hline 15 & 7.30 & 8.60 & 7.30 & 7.20 & 8.40 & 7.00 \\
\hline 30 & 9.90 & 10.60 & 9.70 & 9.80 & 10.40 & 9.72 \\
\hline 45 & 7.20 & 8.55 & 6.88 & 6.80 & 8.50 & 6.75 \\
\hline 60 & 5.20 & 5.40 & 4.80 & 5.30 & 5.45 & 4.65 \\
\hline $\mathrm{CD}$ & 0.083 & 0.053 & 0.069 & 0.067 & 0.059 & 0.070 \\
\hline SE(d) & 0.037 & 0.024 & 0.031 & 0.030 & 0.026 & 0.031 \\
\hline $\mathrm{SE}(\mathrm{m})$ & 0.026 & 0.017 & 0.022 & 0.021 & 0.019 & 0.022 \\
\hline $\mathrm{CV}$ & 0.763 & 0.437 & 0.657 & 0.624 & 0.492 & 0.675 \\
\hline \multirow{3}{*}{$\begin{array}{l}\text { Storage } \\
\text { Period } \\
\text { (Days) }\end{array}$} & \multicolumn{6}{|c|}{ Inoculum Concentration (15\%) } \\
\hline & \multicolumn{3}{|c|}{ IARI Strain } & \multicolumn{3}{|c|}{ IARI Strain } \\
\hline & D & $\mathbf{C}$ & G & D & $\mathbf{C}$ & G \\
\hline 0 & 00.00 & 00.00 & 00.00 & 00.00 & 00.00 & 00.00 \\
\hline 15 & 7.50 & 8.80 & 7.40 & 7.40 & 8.60 & 7.00 \\
\hline 30 & 9.98 & 10.70 & 9.80 & 9.90 & 10.50 & 9.72 \\
\hline 45 & 7.22 & 8.55 & 6.88 & 6.85 & 8.60 & 6.75 \\
\hline 60 & 5.00 & 5.40 & 4.80 & 4.98 & 5.20 & 4.65 \\
\hline CD & 0.067 & 0.053 & 0.065 & 0.053 & 0.061 & 0.070 \\
\hline $\mathrm{SE}(\mathrm{d})$ & 0.030 & 0.023 & 0.029 & 0.024 & 0.027 & 0.037 \\
\hline $\mathrm{SE}(\mathrm{m})$ & 0.021 & 0.017 & 0.020 & 0.017 & 0.019 & 0.022 \\
\hline $\mathrm{CV}$ & 0.612 & 0.428 & 0.615 & 0.497 & 0.499 & 0.675 \\
\hline
\end{tabular}

\section{References}

1. Baisya, R.K. (2004). Post-harvest management of fruits and vegetables-a technology management perspective, Indian Food Pack, July-August, 78-82.
2. Bafrncova, P.; Smogrovicova, D.; Salvikova, I.; Patkova, J. and Domeny, Z. (1999). Improvement of gravity ethanol fermentation by media supplementation using $S$. cerevisiae. Biotechnology Letter, 21: 337-341. 
3. Czyhrinciwk, N. (1966). The technology of passion fruit and mango wines. American Journal of Enology and Viticulture, 17: 27-30.

4. Kumar Y.S.; Prakasam R.S. and Reddy O.V.S. (2009). Optimisation of fermentation conditions for mango (Mangifera indica L.) wine production by employing response surface methodology. International Journal of Food Science and Technology, 44: 2320-2327.

5. Onkarayya, H. and Singh, H. (1986). Screening of mango varieties for dessert and madeirastyle wine. American Journal of Enology and Viticulture, 35:63-65.

6. Reddy L.V.A. and Reddy O.V.S. (2005). Production and characterization of wine from mango fruit (Mangifera indica L). World Journal of Microbiology and Biotechnology 21:1345-1350.

7. Varakumar S.; Kumar Y.S. and Reddy O.V.S. (2011). Carotenoid composition of mango (Mangifera indica L.) wine and its antioxidant activity. Journal of Food Biochemistry, 35: 1538-1547.

8. Walker, G.M., (1998). Yeast Physiology and Biotechnology. John Wiley \& Sons, England. 